Title : will be set by the publisher

Editors : will be set by the publisher

EAS Publications Series, Vol. ?, 2006

\title{
CHARACTERISATION OF THE DOME C ATMOSPHERIC BOUNDARY LAYER TURBULENCE WITH A NON-DOPPLER ACOUSTIC RADAR
}

\author{
J.S. Lawrence ${ }^{1}$, M.C.B. Ashley ${ }^{1}$, C.S. Bonner ${ }^{1}$, S. Bradley ${ }^{2}$, D. \\ Luong-Van ${ }^{1}$ and J.W.V. Storey ${ }^{1}$
}

\begin{abstract}
The Antarctic plateau has superb astronomical seeing above a turbulent boundary layer. This layer has a thickness of between tens of metres and a few hundred metres, depending on the site. We are developing a sonic radar, SNODAR, to measure the turbulence in the boundary layer from 10 to 50 metres, and, in particular, to measure the height of the boundary layer to an accuracy of 1 metre. Commercial sonic radars typically have a lower limit of about 10 metres, and have 10 metre range bins. The results from SNODAR should allow a confident assessment of the height at which one must mount a telescope in order to realise the superb free atmosphere seeing from the Antarctic plateau, which has been measured at Dome $\mathrm{C}$ to be $\sim 0.3$ arcsecs.
\end{abstract}

\section{Introduction}

Winter-time data from Dome C station on the Antarctic plateau have demonstrated exceptionally calm atmospheric conditions above a strongly turbulent surface boundary layer 30-35 m thick (Lawrence et al. 2004; Agabi et al. 2006). It is now imperative to obtain long-term, high-temporal resolution, high-spatial resolution data on the variability, height, and distribution of this turbulent surface layer. This is difficult to achieve with existing turbulence profiling techniques, such as tower-mounted or balloon-borne sonic anemometers or microthermal probes, MASS and SCIDAR scintillation-based instruments, or commercially available sonic radars (SODAR). We are developing a new instrument called SNODAR (Surface-layer NOn Doppler Acoustic Radar) which uses separate transmit and receive piezo-electric horn arrays to measure the acoustic signal backscattered by atmospheric turbulence in the near-surface layer.

\footnotetext{
${ }^{1}$ School of Physics, University of New South Wales, Sydney, NSW 2052 Australia; e-mail: jl@phys. unsw. edu. au

${ }^{2}$ Faculty of Science, University of Auckland, Auckland, New Zealand
} 

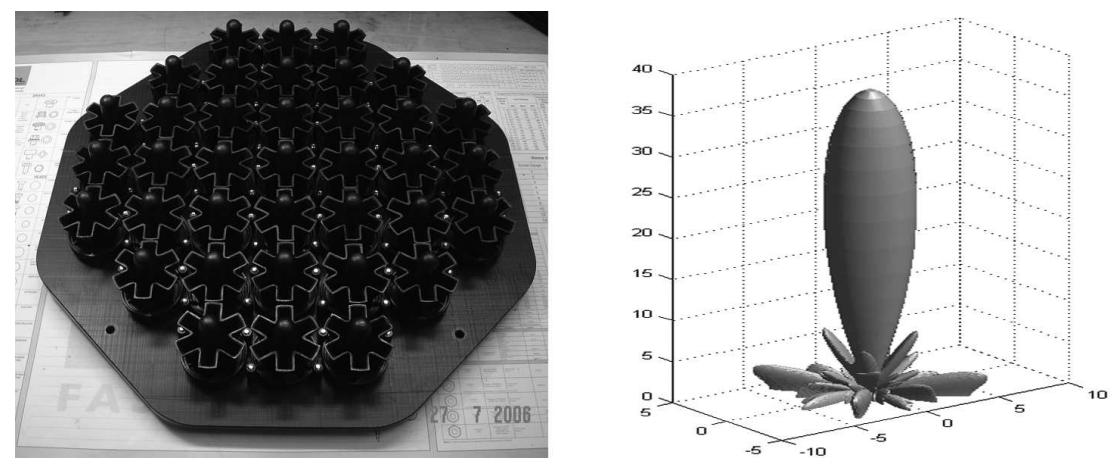

Fig. 1. Left: prototype array of 37 piezo-electric horns. Right: calculated 3D beam profile of the prototype array at $5 \mathrm{kHz}$.

\section{Acoustic Design and Sensitivity}

The baseline design for SNODAR consists of 2 separate 37 element phased arrays of piezoelectric horns. The arrays are each $400 \mathrm{~mm}$ in diameter and are separated by $50 \mathrm{~mm}$. Using a total electrical input power of $100 \mathrm{~W}$, and a sensitivity of 94 dB SPL per Watt per speaker, we can produce a beam of $4 \mathrm{~W}$ acoustic power with a $10^{\circ}$ divergence. Fig. 1 shows a prototype array and the calculated beam profile. Acoustic baffling will be installed around each array to reduce interference from side lobes and ground clutter.

The transmit array emits an acoustic pulse with a pulse length of $6.7 \mathrm{~ms}$, which is appropriate to obtain a spatial resolution of $1 \mathrm{~m}$. The receive array measures the backscattered acoustic power over time, $P_{r}(t)$, which is directly proportional to $C_{T}^{2}(z)$, the atmospheric temperature structure constant as a function of height (see e.g., Tartarski 1961). The $C_{T}^{2}(z)$ profile is closely related to the refraction index structure constant profile, $C_{N}^{2}(z)$, which characterises the effects of turbulence on optical and infrared imaging, and can be used to derive the atmospheric seeing. The height-dependent proportionality constant (between $P_{r}$ and $C_{T}^{2}(z)$ ) depends on the scattering cross-section and the attenuation of sound in air, both of which scale with acoustic frequency. For a height of $50 \mathrm{~m}$ with typical mid-winter Dome $\mathrm{C}$ meteorological conditions, the optimum frequency which maximises the return power is in the range $5-6 \mathrm{kHz}$. Fig. 2 shows the expected values of $C_{T}^{2}(z)$ within the near-surface layer at Dome $\mathrm{C}$ during winter (derived from Agabi et al. 2006). The expected received voltage per pulse for SNODAR is also shown for a $5 \mathrm{kHz}$ signal with a $6.7 \mathrm{~ms}$ (34 cycle) pulse length, compared to the $1 \mathrm{nV} . \mathrm{Hz}^{-0.5}$ op-amp electronic noise. A $S N R>1$ per pulse is obtained up to a height of $100 \mathrm{~m}$ above the surface. It is likely that the ultimate sensitivity limit per pulse will be due to background ambient noise. This noise level is difficult to estimate (Krasnenko \& Odintsov 1984) but is expected to be equal to the backscattered signal at a height of 50-80 m. It should be possible to increase the $S N R$ via time series 

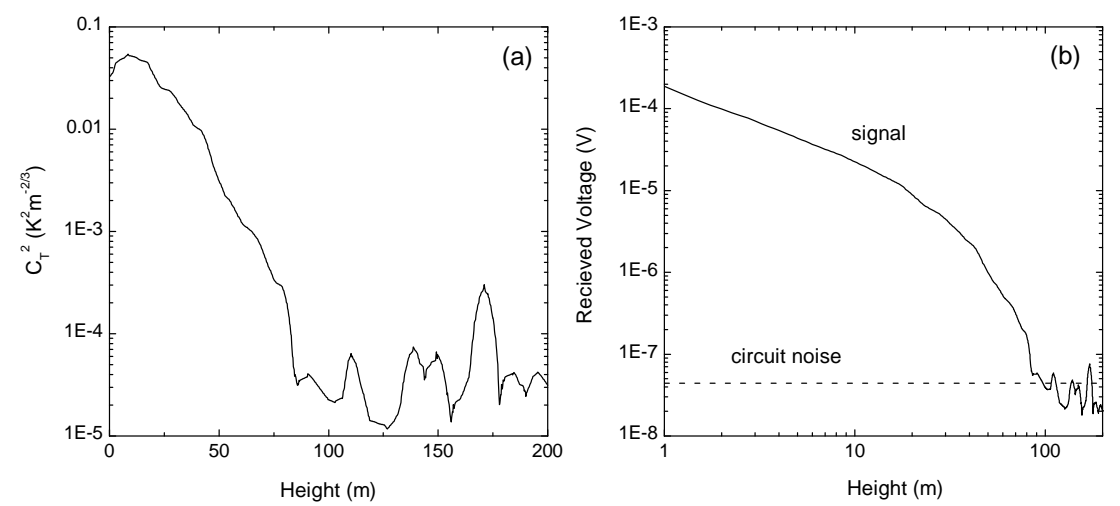

Fig. 2. (a) Temperature structure function profile of the Dome $\mathrm{C}$ surface layer measured by microthermal balloons. (b) Estimated received signal voltage for a $6.7 \mathrm{~ms} 5 \mathrm{kHz}$ acoustic pulse compared to the op-amp noise in a $2 \mathrm{kHz}$ bandwidth.

averaging and digital processing techniques. Previous acoustic radar experiments at Dome C (Travouillon et al. 2003; Argentini et al. these proceedings) have confirmed that the ambient noise level is very low.

The minimum height of the first backscattered signal measurable with an acoustic radar is determined by the natural resonances (or ringing) of the speaker system used. The magnitude and decay time of this resonance typically renders data from the first few tens of metres useless. We are currently investigating designs for SNODAR that will reduce this resonance with the aim of measuring turbulence down to the first $5 \mathrm{~m}$ above ground level.

\section{Electronics and Control System}

SNODAR consists of an external acoustic antenna array and an internally housed data- acquisition and control system, as shown in Fig. 3. A PC104 stacking SingleBoard-Computer running Linux is used for instrument control, data collection and analysis, and communications. The output audio pulse duration, shape, and magnitude is controlled via a 24-bit $96 \mathrm{kHz}$ USB Audio IO device, which drives a 300 $\mathrm{W}$ audio amplifier connected to the transmit array. The receive array elements are connected in parallel to a co-located ultra-low noise summing pre-amplifier stage with a $2 \mathrm{kHz}$ bandwidth analog bandpass filter. The amplified signal is then sent back to the laboratory building, where it is further amplified by a programmable gain stage before being digitised by the Audio card. A set of shell and C programs control audio output configuration, data collection routines, and data analysis. All data will be stored locally on a solid- state hard disk. SNODAR will be able to communicate via Ethernet to a local computer with Iridium Satellite Network communication capabilities. This low bandwidth connection allows lim- 


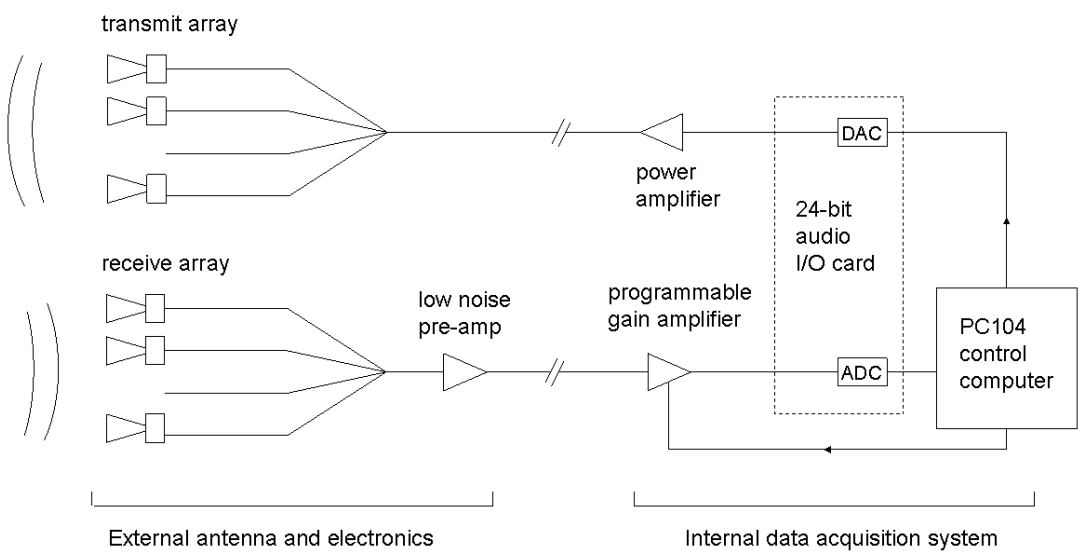

Fig. 3. SNODAR system schematic.

ited instrument and housekeeping data downloads, and instrument control system modification and upgrades.

\section{Conclusion}

We have described here the design for an instrument, SNODAR, to measure the boundary layer turbulence at Antarctic plateau sites with high spatial and high temporal resolution. Sensitivity calculations show that the baseline design should achieve approximately $1 \mathrm{~m}$ spatial resolution to at least $50 \mathrm{~m}$ above the surface. The expected noise performance has been confirmed with a prototype speaker array. Further testing and design of this instrument will continue before a planned deployment to Dome C and/or Dome A over the 2007/8 austral summer.

\section{References}

Agabi, A., Aristidi, E., Azouit, M., Fossat, E., Martin, F., Sadibekova, T., Vernin, J., \& Ziad, A. 2006, PASP, 118, 344

Krasnenko, N. P., \& Odintsov, S.L. 1984, J. Acoust. Soc. Am., 75, 390

Lawrence, J. S., Ashley, M. C. B., Travouillon, T. \& Tokovinin, A. 2004, Nat, 431, 278

Tatarski, V.I., 1961, Wavefront Propagation in a Turbulent Medium, McGraw-Hill, New York

Travouillon, T., Ashley, M.C.B., Burton, M.G., Storey, J.W.V. \& Loewenstein, R.F. 2003. A\&A, 400, 1163 